\title{
NONLINEAR DIFFERENTIAL EQUATIONS AND ALGEBRAIC SYSTEMS
}

\author{
LLOYD K. WILLIAMS \\ Department of Mathematics \\ Texas Southern University \\ Houston, Texas 77004 \\ U.S.A.
}

(Received April 17, 1978)

ABSTRACT. In this paper we obtain the general solution of scalar, first-order differential equations. The method is variation of parameters with asymptotic series and the theory of partial differential equations.

The result gives us a form like a differential quotient requiring only that a limit be taken. Like the familiar expression for the solution of linear, first order, ordinary equations, it is the same in all cases.

KEY WORDS AND PHRASES. Riccati Equations, Abel Equations, Cauchy-Kowalewski Theorem, Cauchy-Kowalewski System, Universal Cauchy-Kowalewski System.

AMS (MOS) SUBJECT CLASSIFICATION (1970) CODES. 34A0S, 34A10, $34 A 15$.

1. INTRODUCTION.

We present a unified treatment for the general scalar, first-order, ordinary differential equation

$$
y^{\prime}=G(x, y), G \in C^{I} \text {. }
$$


Particular examples are linear equations, Riccati equations and Abel equations.

\section{PRELIMINARIES.}

We begin with the differential system

$$
\left\{\begin{array}{l}
\mathrm{v}_{1}^{\prime}=\mathrm{f}\left(\mathrm{v}_{1}, \mathrm{v}_{2}\right)=-\mathrm{v}_{1} \mathrm{v}_{2} \\
\mathrm{v}_{2}^{\prime}=\mathrm{h}\left(\mathrm{v}_{1}, \mathrm{v}_{2}\right)=\mathrm{v}_{1}-\mathrm{v}_{2} \\
\mathrm{v} \neq 0
\end{array}\right\}
$$

with general solution $v_{1}=v_{1}\left(x, c_{1}, c_{2}\right), v_{2}=v_{2}\left(x, c_{1}, c_{2}\right)$. Here $c_{1}, c_{2}$ are arbitrary constants.

Now let $x=x(t)$. Then we get

$$
\left\{\begin{array}{l}
\dot{\mathrm{v}}_{1}=\mathrm{u}_{1} \dot{\mathrm{x}} \quad \cdot=\frac{\mathrm{d}}{\mathrm{dt}} \\
\mathrm{v}_{2}=\mathrm{U}_{2} \dot{\mathrm{x}} \\
\mathrm{U}_{1}=\mathrm{f}\left(\mathrm{v}_{1}, \mathrm{v}_{2}\right), \mathrm{u}_{2}=\mathrm{h}\left(\mathrm{v}_{1}, \mathrm{v}_{2}\right) \\
\mathrm{v}_{1} \neq 0
\end{array}\right\}
$$

We are now ready to present the algebraic system referred to in the title.

\section{THE CAUCHY-KOWALEWSKI SYSTEM.}

Let $w_{1}=w_{1}(t, \varepsilon), w_{2}=w_{2}(t, \varepsilon)$ be two functions of $t$ and $\varepsilon$ (at present unknown).

The functions $v_{1}, v_{2}$ have been given by (2.1). Finally two more unknown functions $K\left(w_{1}, w_{2}, t, \varepsilon\right)$ and $L\left(w_{1}, w_{2}, t, \varepsilon\right)$ will be defined by partial differential equations later. They will contain another variable, $\lambda$. It will be possible to substitute an arbitrary $G\left(w_{1}, t\right)$ for $\lambda$ to solve specific equations. 
DEFINITION. The system of algebraic equations

$$
\left\{\begin{array}{cc}
\text { (a) } \mathrm{w}_{1}-\mathrm{K}\left(\mathrm{w}_{1}, \mathrm{w}_{2}, t, \varepsilon\right) \mathrm{v}_{1}=0 \\
\text { (b) } \mathrm{w}_{2}^{2}-\mathrm{L}\left(\mathrm{w}_{1}, \mathrm{w}_{2}, t, \varepsilon\right)-\mathrm{v}_{2}=0 \\
\text { (c) } \quad \mathrm{x}=\mathrm{w}_{1}+\mathrm{tw}_{2} . \\
\mathrm{w}_{1} \neq 0
\end{array}\right\}
$$

is called the Cauchy-Kowalewski system, for a specific $G\left(w_{1}, t\right)$. Using $\lambda$ we will get a universal system.

Under suitable conditions on the functions $K$ and $L$, we can solve it for $w_{1}=w_{1}(t, \varepsilon)$ and $w_{2}=w_{2}(t, \varepsilon)$. We proceed by defining these functions as solutions of appropriate partial differential equations. We will derive these functions $L\left(w_{1}, w_{2}, t, \varepsilon, \lambda\right)$ and $K\left(w_{1}, w_{2}, t, \varepsilon, \lambda\right)$ and regard them as fixed like universal constants.

\section{THE FIRST FUNCTION K IN THE CAUCHY-KOWALEWSKI SYSTEM.}

We differentiate $3(a-b)$ with respect to $t$ to get expressions for $\dot{w}_{1}, \dot{w}_{2}$. Denoting the expression for $\dot{\mathrm{w}}_{1}$ by $\mathrm{R}$ we get

$$
\dot{\mathrm{w}}_{1}=\mathrm{R}
$$

To simplify notation, let $K=\alpha$ in (4.1) and get

$$
\dot{w}_{1}=R=\frac{A_{1} L_{2}+A_{2} L_{3}+A_{3}}{-A_{2} L_{1}+A_{4} L_{2}+A_{5}}
$$

Some of the $A_{i}, i=1, \ldots, 5$ are given explicitly later. These are not partial derivatives. By contrast,

$$
\mathrm{L}_{1}=\frac{\partial \mathrm{L}}{\partial \mathrm{w}_{1}} \text { etc. }
$$

Now let $z=L-w_{2}^{2}$ and note that from $(2.1), 3(a-b)$ we have $f=\frac{w_{1}}{\alpha} z$, $h=\frac{w_{1}}{\alpha}+z$ in the new notation. 
The following equation is of fundamental importance. We arbitrarily set

$$
A_{2}=2 w_{1} w_{2} \alpha_{1}-w_{1} t h \alpha_{1}+w_{1} \alpha_{3}+t f \alpha^{2}=\varepsilon
$$

where $\mathrm{K}=\alpha=\alpha\left(\mathrm{L}, \mathrm{w}_{1}, \mathrm{w}_{2}, \mathrm{t}, \varepsilon\right)$ and $\alpha_{1}=\frac{\partial \alpha}{\partial \mathrm{L}}$, etc., for real $\varepsilon>0$.

By the Cauchy-Kowalewski theorem [See e.g. (2.1)] let $\alpha_{0}=\alpha_{0}\left(L, w_{1}, w_{2}, t, \varepsilon\right)$ be an analytic solution of (4.2). Further, we will write

$$
\bar{A}_{i}=A_{i}\left(\alpha_{0}\right), \quad i=1,2,3,4,5 .
$$

Let $\alpha_{0}=\sum_{n=0}^{\infty} c_{n} \varepsilon^{n}$ where $c_{n}=c_{n}\left(L, w_{1}, w_{2}, t\right)$ are analytic. Before imposing conditions on $c_{0}$ we give the following definitions.

DEFINITION. $\underset{\varepsilon \rightarrow 0}{\operatorname{L}}\left[\left(\frac{w_{1}}{\alpha}+z\right)\left(w_{1} \alpha_{04}-w_{1} w_{2} \alpha_{02}+w_{2} \alpha_{0}\right)\right]=s_{1}\left(L, w_{1}, w_{2}, t\right)$.

Two more of the $\bar{A}_{i}$ will now be given explicitly.

$$
\begin{aligned}
& \bar{A}_{1}=\left(\frac{w_{1}}{\alpha_{0}}+z\right)\left(w_{1} \alpha_{04}-w_{1} w_{2} \alpha_{02}+w_{2} \alpha_{0}\right) \\
& \bar{A}_{4}=w_{1} \alpha_{02}+\alpha_{0}^{2} f-\alpha_{0}-w_{1} h \alpha_{01}
\end{aligned}
$$

DEFINITION. $\underset{\varepsilon \rightarrow 0}{\mathrm{~L}} \overline{\mathrm{A}}_{1}=\Delta$.

DEFINITION. $\underset{\varepsilon \rightarrow 0}{L}\left(\bar{A}_{1}-G\left(w_{1}, t\right) \bar{A}_{4}\right)=S_{2}\left(L, w_{1}, w_{2}, t\right)$.

The conditions on $c_{0}$ can be stated now as follows:
(1) $c_{0} \neq 0$,
(2) $\mathrm{s}_{1}\left(\mathrm{~L}, \mathrm{w}_{1}, \mathrm{w}_{2}, t\right) \neq 0$,
(3) $\Delta \neq 0$.

Substituting $\alpha_{0}=\sum_{n=0}^{\infty} c_{n} \varepsilon^{n}$ in (4.2) we get

$$
2 w_{1} w_{2} c_{01}-w_{1} t\left(\frac{w_{1}}{c_{0}}+z\right) c_{01}+w_{1} c_{03}+t w_{1} z c_{0}=0
$$


of which some solutions are given

$$
\mathrm{H}\left[\beta\left(\mathrm{c}_{\mathrm{o}}, \mathrm{z}, \mathrm{w}_{1}\right), \mathrm{w}_{2}+\frac{1}{\mathrm{t}} \mathrm{P}\left(\mathrm{c}_{\mathrm{o}}, \mathrm{w}_{1}, \beta\left(\mathrm{c}_{\mathrm{o}}, \mathrm{z}, \mathrm{w}_{1}\right)\right)\right]=\text { constant }
$$

where

(1) $\mathrm{H}$ is arbitrary

(2) $\beta$ satisfies the partial differential equation

$$
\begin{aligned}
& c_{0} z \beta_{1}+\left(\frac{w_{1}}{c_{0}}+z\right) \beta_{2}=0 \\
& \left(w_{1} \beta_{3}+c_{0} \beta_{1} \neq 0\right)
\end{aligned}
$$

(3) $P$ is defined as follows: first solve $B\left(c_{0}, w_{1}, z\right)=a$ for $z=Q\left(c_{0}, w_{1}, a\right)$. Then set

$$
P=\int \frac{d c_{0}}{c_{0} Q\left(c_{0}, w_{1}, a\right)} .
$$

THEOREM 1. The function $\mathrm{H}$ can be chosen analytic in (4.3a) so that conditions (2.1), (2.2), (3.1) hold for $c_{0}$.

PROOF. Let $\gamma=w_{2}+\frac{1}{t} P$ and then $(4.3 a)$ becomes $H(\beta, \gamma)=$ constant. The partial derivatives of $c_{0}$ are computed from (4.3a) and from them we see that $\mathrm{H}_{\gamma} \neq 0$ implies that $\frac{\partial c_{\mathrm{O}}}{\partial t} \neq 0$, so condition (2.1) holds. Further, $\Delta=\underset{\varepsilon \rightarrow 0}{\mathrm{~L}} \overline{\mathrm{A}}_{1}=0$ implies $\left(\frac{P}{t}+w_{2}\right) H_{\gamma}=0$. So $H_{\gamma} \neq 0$ implies $\Delta \neq 0$. Thus (2.1), (2.2) hold if merely $H_{\gamma} \neq 0$. Now $S_{1}=0$ implies that $t_{2}\left(w_{1} \beta_{3}+c_{o} \beta_{1}\right) H_{B}+H_{\gamma}=0$. Since $w_{1} \beta_{3}+c_{o} \beta_{1} \neq 0$, we can choose $H$ so that $s_{1} \neq 0$. This completes the proof. Summarizing the results of this section, $K=\alpha=\alpha_{0}$ can be defined as the solution of (4.2) where $\mathrm{H}$ is analytic, $c_{0} \neq 0, \mathrm{~S}_{1} \neq 0$, and $\Delta \neq 0$. To solve (3.1) however, we must define L.

5. SOLUTION OF THE CAUCHY-KOWALEWSKI SYSTEM.

To solve the system (3.1), we must now define the function $L\left(w_{1}, w_{2}, t, \varepsilon\right)$. 
Setting $\dot{\mathrm{w}}=\mathrm{G}, \alpha=\alpha_{0}$ and $\overline{\mathrm{A}}_{2}=\varepsilon,(4.2)$ in (4.1a) suggests defining $\mathrm{L}$ by

$$
\varepsilon \mathrm{GL}_{1}+\left(\overline{\mathrm{A}}_{1}-\overline{G A}_{4}\right) \mathrm{L}_{2}+\varepsilon \mathrm{L}_{3}=\overline{G A}_{5}-\overline{\mathrm{A}}_{3} \text {. }
$$

$L_{1}=\frac{\partial L}{\partial w_{1}}$, etc. This does not seem to be feasible. Instead, letting $\varepsilon$ tend to zero leads to

$$
L_{2}=\frac{\partial L}{\partial w_{2}}=\frac{\overline{G A}_{5}-\bar{A}_{3}}{\bar{A}_{1}-G \bar{A}_{4}}
$$

This will be used to define L.

Let $\lambda$ be a new variable and consider

$$
\mathrm{L}_{2}=\frac{\lambda \overline{\mathrm{A}}_{5}-\overline{\mathrm{A}}_{3}}{\overline{\mathrm{A}}_{1}-\lambda \overline{\mathrm{A}}_{4}}
$$

Note that the right side of (5.2) is analytic where $w_{1} \neq 0$ and $\bar{A}_{1}-\lambda \bar{A}_{4} \neq 0$. So let $L=\bar{L}\left(w_{1}, w_{2}, t, \varepsilon, \lambda\right)=P_{1}\left(w_{2}\right)+P_{2}\left(w_{1}, w_{2}, t, \varepsilon, \lambda\right)$ be an analytic solution on (5.2) and assume that none of the expressions $\Delta, \mathrm{s}_{1}, \mathrm{c}_{0}$ vanish when $L \equiv P_{1}\left(w_{2}\right)$.

Now since the value of $\frac{\partial}{\partial w_{2}}\left(\bar{L}\left(w_{1}, w_{2}, t, \varepsilon, \lambda\right)\right.$ for $\lambda=G\left(w_{1}, t\right)$ is the same as $\frac{\partial}{\partial \mathrm{w}_{2}}\left(\overline{\mathrm{L}}\left(\mathrm{w}_{1}, \mathrm{w}_{2}, t, \varepsilon, G\left(\mathrm{w}_{1}, t\right)\right)\right.$ we see that $\overline{\overline{\mathrm{L}}}\left(\mathrm{w}_{1}, \mathrm{w}_{2}, t, \varepsilon\right) \equiv \overline{\mathrm{L}}\left(\mathrm{w}_{1}, \mathrm{w}_{2}, t, \varepsilon, G\left(\mathrm{w}_{1}, t\right)\right)$ is a solution of (5.1) for any $G$. Moreover $\overline{\bar{L}} \varepsilon C^{I}$ since $G$ is continuous and $\bar{L}$ is analytic. Let $K_{G}=\alpha_{0}\left(\overline{\overline{\mathrm{L}}}, \mathrm{w}_{1}, \mathrm{w}_{2}, t\right)$ and $L=\overline{\overline{\mathrm{L}}}$.

We now prove the solvability near suitable points of the Cauchy-Kowalewski system. The variable $\lambda$ gives our functions the universal character referred to previously.

LEMMA I. Let $(a, b, c)$ be such that $S_{1}\left(P_{1}(b) a, b, c\right) \neq 0$. Then, for small $t$, the Jacobian of (3.1) is nonzero at $(a, b, c, \varepsilon)$. 
PROOF. If the Jacobian of $(3.1)=0$, then

$$
-\overline{\mathrm{A}}_{2} \mathrm{~L}_{1}+\overline{\mathrm{A}}_{4} \mathrm{~L}_{2}+\overline{\mathrm{A}}_{5}=0
$$

The subsidiary equations of (5.3) are:

$\frac{d w_{1}}{-\bar{A}_{2}}=\frac{d w_{2}}{\bar{A}_{4}}=\frac{d L}{-\bar{A}_{5}}$, so that $\frac{d L}{d w_{2}}=\frac{-\bar{A}_{5}}{\bar{A}_{4}}$

But from (5.1), $\frac{\mathrm{dL}}{\mathrm{dw}}=\frac{\mathrm{GA}_{5}-\overline{\mathrm{A}}_{3}}{\overline{\mathrm{A}}_{1}-\overline{G A}_{4}}$

Thus $\overline{\mathrm{A}}_{1} \overline{\mathrm{A}}_{5}-\overline{\mathrm{A}}_{3} \overline{\mathrm{A}}_{4}=0$.

But $\bar{A}_{1} \bar{A}_{5}-\bar{A}_{3} \bar{A}_{4}=\left(w_{1} \alpha_{04}-w_{1} w_{2} \alpha_{02}+w_{2} \alpha_{0}\right)\left(\frac{w_{1}}{\alpha_{0}}+z\right) \varepsilon$. So

$\underset{\varepsilon \rightarrow 0}{L}\left(w_{1} \alpha_{04}-w_{1} w_{2} \alpha_{02}+w_{2} \alpha_{0}\right)\left(\frac{w_{1}}{\alpha_{0}}+z\right)=0$. However

$\underset{\varepsilon \rightarrow 0}{L}\left(w_{1} \alpha_{04}-w_{1} w_{2} \alpha_{02}+w_{2} \alpha_{0}\right)\left(\frac{w_{1}}{\alpha_{0}}+z\right)=s_{1}\left(P_{1}\left(w_{2}\right), w_{1}, w_{2}, t\right) \neq 0$ and the proof is complete.

We next consider continuity in order to apply the implicit function theorem to (3.1). We first observe that $\underset{\varepsilon \rightarrow 0}{L} \bar{A}_{1} \neq 0$. If $\underset{\varepsilon \rightarrow 0}{L} \bar{A}_{4}=0$, then $\underset{\varepsilon \rightarrow 0}{L}\left(\bar{A}_{1}-\overline{G A}_{4}\right) \neq 0$.

Now consider the case where $\underset{\varepsilon \rightarrow 0}{\operatorname{L}} \bar{A}_{4} \neq 0$, but $\underset{\varepsilon \rightarrow 0}{\operatorname{L}}\left(\bar{A}_{1}-G \bar{A}_{4}\right)=0$.

LEMMA II. There is at most one function $G$ such that $\underset{\varepsilon \rightarrow 0}{L}\left(\bar{A}_{1}-G \bar{A}_{4}\right)=0$.

PROOF. $\overline{\bar{L}}\left(w_{1}, w_{2}, t, \varepsilon\right)=\bar{L}\left(w_{1}, w_{2}, t, G\left(w_{1}, t\right)\right)=P_{1}\left(w_{2}\right)+\varepsilon P_{2}\left(w_{1}, w_{2}, t, \varepsilon, G\left(w_{1}, t\right)\right)$. So it and its partials with respect to $w_{1}, w_{2}, t$ do not contain $G$ as $\varepsilon \rightarrow 0$. Since $\alpha_{0}=\sum_{n=0}^{\infty} c_{n}\left(L, w_{1}, w_{2}, t\right)=c_{0}\left(L, w_{1}, w_{2}, t\right)+c_{1}\left(L, w_{1}, w_{2}, t\right)+c_{2}\left(L, w_{1}, w_{2}, t\right) \varepsilon^{2}+\ldots$, 
the same holds for it.

Thus $\underset{\varepsilon \rightarrow 0}{\mathrm{~L}} \bar{A}_{1}$ and $\underset{\varepsilon \rightarrow 0}{\mathrm{~L}} \bar{A}_{4}$ are independent of $G$.

So $G=\frac{\underset{\varepsilon \rightarrow 0}{L} \bar{A}_{1}}{\underset{\varepsilon \rightarrow 0}{L} \bar{A}_{4}}$. This completes the proof.

In the sequel, we fgnore this possible exception and assume that $\underset{\varepsilon \rightarrow 0}{L}\left(\bar{A}_{1}-\overline{G A}_{4}\right) \neq 0$ for any $G$.

LEMMA III. If $(a, b, c)$ is such that $S_{2}\left(P_{1}(b), a, b, c\right) \neq 0$, there is an $\varepsilon>0$ such that the left sides of (3.1) are $C^{I}$ at $(a, b, c, \varepsilon)$.

PROOF. Based on analytic properties of $\mathrm{v}_{1}, \mathrm{v}_{2}, \overline{\overline{\mathrm{L}}}, \mathrm{K}_{\mathrm{G}}$ and the nonvanishing of $\mathrm{S}_{2}$, we will not give details.

Choosing constant values for $w_{1}, w_{2}$ in (3.1), we can get $c_{1}(\varepsilon), c_{2}(\varepsilon)$ so that left sides vanishes and apply the implicit function theorem to (3.1). Then we solve for $w_{1}(t, \varepsilon)$ and $w_{2}(t, \varepsilon)$. Here $c_{1}, c_{2}$ come from equation (2.1) of section 2 .

\section{THE PRINCIPAL DIFFERENTIAL EQUATION.}

We now consider the differential equation

$$
\frac{d y}{d x}=y^{\prime}=g(x, y)
$$

DEFINITION. $\mathrm{W}_{1}(t)=\underset{\varepsilon \rightarrow 0}{\mathrm{~L}} \mathrm{w}_{1}(t, \varepsilon)$.

It will be shown that $W_{1}(t)$ satisfies (6.1). Of course we change $y, x$ to $W_{1}, t$ respectively.

We begin this process with

THEOREM II. Let $S_{1} \neq 0$ at $\left(\bar{w}_{1}, \bar{w}_{2}, \bar{t}\right)$. Then $\frac{d}{d t} w_{1}(t, \varepsilon) \rightarrow G\left(\bar{w}_{1}, \bar{t}\right)$ as $\varepsilon \rightarrow 0$.

PROOF. $\overline{\bar{L}}=P_{1}\left(w_{2}\right)+\varepsilon P_{2}\left(w_{1}, w_{2}, t, G\left(w_{1}, t\right)\right)$ so that $\frac{\partial \overline{\bar{L}}}{\partial w_{1}} \rightarrow 0$ as $\varepsilon \rightarrow 0$ and also $\frac{\partial \overline{\bar{L}}}{\partial t} \rightarrow 0$ as $\varepsilon \rightarrow 0$. Thus $\overline{\overline{\mathrm{L}}}_{1}, \overline{\overline{\mathrm{L}}}_{3} \rightarrow 0$ as $\varepsilon \rightarrow 0$. 
Now (5.1) $\bar{A}_{1} L_{2}+\bar{A}_{3}=G\left(\bar{A}_{4} L_{2}+\bar{A}_{5}\right)$.

Also $\bar{A}_{4} L_{2}+\bar{A}_{5}=\frac{\bar{A}_{1} \bar{A}_{5}-\bar{A}_{3} \bar{A}_{4}}{\bar{A}_{1}-\overline{G A}_{4}}=\frac{S_{1} \varepsilon}{\bar{A}_{1}-G \bar{A}_{4}}$.

Thus $R=\frac{\bar{A}_{1} L_{2}+\bar{A}_{2} L_{3}+\bar{A}_{3}}{-\bar{A}_{2} L_{1}+\bar{A}_{4} L_{2}+\bar{A}_{5}}=\frac{\varepsilon L_{3}+\bar{A}_{1} L_{2}+\bar{A}_{3}}{-\varepsilon L_{1}+\bar{A}_{4} L_{2}+\bar{A}_{5}}$.

So $\quad \dot{w}_{1}=\frac{\varepsilon L_{3}+G\left(\bar{A}_{4} L_{2}+\bar{A}_{5}\right)}{-\varepsilon L_{1}+\left(\bar{A}_{4} L_{2}+\bar{A}_{5}\right)}=\frac{\left(\bar{A}_{1}-\overline{A A}_{4}\right) L_{3}+G S_{1}}{-\left(\bar{A}_{1}-\overline{G A}_{4}\right) L_{1}+S_{1}}$.

Therefore $\dot{\mathrm{w}}_{1} \rightarrow \frac{\mathrm{GS}_{1}}{\mathrm{~S}_{1}}$ as $\varepsilon \rightarrow 0$ and $\mathrm{S}_{1} \neq 0$. This completes the proof.

By the last theorem, $\underset{\varepsilon \rightarrow 0}{L} \frac{d}{d t} w_{1}(t, \varepsilon)=\underset{\varepsilon \rightarrow 0}{L} G\left(w_{1}(t, \varepsilon), t\right)=G\left(\underset{\varepsilon \rightarrow 0}{L} w_{1}(t, \varepsilon), t\right)=$ $G\left(W_{1}(t), t\right)$.

But also it is true [2: P.461] that

$$
\underset{\varepsilon \rightarrow 0}{L} \frac{d}{d t} w_{1}(t, \varepsilon)=\frac{d}{d t}\left(\underset{\varepsilon \rightarrow 0}{L} w_{1}(t, \varepsilon)\right)=W_{1}^{\prime}(t) .
$$

So

$$
W_{1}^{\prime}(t)=G\left(W_{1}(t), t\right)
$$

7. PARTICULAR AND GENERAL SOLUTIONS OF $y^{\prime}=G(x, y)$.

7 (a) PARTICULAR SOLUTIONS. Let $J\left(w_{1}, t\right) \varepsilon C^{I}$, $L^{*}\left(w_{1}, w_{2}, t\right)=\bar{L}\left(w_{1}, w_{2}, t, \varepsilon, J\left(w_{1}, t\right)\right)$ and $\alpha^{*}\left(w_{1}, w_{2}, t\right)=\alpha_{0}\left(L^{*}, w_{1}, w_{2}, t\right)$.

Let $Q$ be the set of points in $\left(w_{1}, w_{2}, t\right)$-space where
(1) $w_{1} \neq 0$
(2) $c_{0} \neq 0$
(3) $s_{1} \neq 0$
(4) $s_{2} \neq 0$.

Let $\bar{Q}$ be the projection of $Q$ on the $\left(w_{1}, t\right)$ plane.

The Universal Cauchy-Kowalewski System DEFINITION. $\bar{\alpha}\left(w_{1}, w_{2}, t, \varepsilon, \lambda\right)=\alpha_{0}\left(\bar{L}, w_{1}, w_{2}, t\right)$.

DEFINITION. $F_{1} \equiv w_{1}-\bar{\alpha} v_{1}\left(w_{1}+t w_{2}, c_{1}, c_{2}\right)$. 
DEFINITION. $\mathrm{F}_{2} \equiv \mathrm{w}_{2}^{2}-\overline{\mathrm{L}}\left(\mathrm{w}_{1}, \mathrm{w}_{2}, t, \varepsilon, \lambda\right)-\mathrm{v}_{2}\left(\mathrm{w}_{1}+\mathrm{tw}, \mathrm{w}_{2}, \mathrm{c}_{2}\right)$.

DEFINITION. $F_{3} \equiv \bar{A}_{1}-J\left(w_{1}, t\right) \bar{A}_{4}$ with $\lambda$ replaced by $J\left(w_{1}, t\right)$.

DEFINITION. The system $\left\{\begin{array}{l}F_{1}=0 \\ F_{2}=0 \\ F_{3} \neq 0\end{array}\right\}$ is also called the Universal

Cauchy-Kowalewski System.

We refer to it in the following

THEOREM III. Let $P \in \bar{Q}$. There is a region in which the solution through $P$ of $\dot{w}_{1}=J\left(w_{1}, t\right)$ is determined as follows:

(1) In $F_{1}, F_{2}$ replace $\lambda$ by $J\left(w_{1}, t\right)$ and $c_{1}, c_{2}$ by suitable functions of $\varepsilon$.

(2) Equate the results in (2.1) to zero.

(3) Solve the resulting system for $w_{1}(t, \varepsilon)$ and $w_{2}(t, \varepsilon)$.

(4) Take the limit of $\mathrm{w}_{1}(t, \varepsilon)$ as $\varepsilon \rightarrow 0$.

PROOF. Let $P=\left(a, t_{0}\right), P \in \bar{Q}$. Since $c_{0}\left(P_{1}(b), a, b, t_{0}\right) \neq 0$, there is an $\varepsilon$ such that $\alpha^{*}\left(a, b, t_{0} \varepsilon\right) \neq 0$. Let $\left(\bar{v}_{1}, \bar{v}_{2}\right)$ be a solution of (2.1) such that

$$
\left\{\begin{array}{l}
\bar{v}_{1}\left(a+t_{0} b\right)=\frac{a}{a^{*}\left(a, b, t_{0}, \varepsilon\right)} \\
\bar{\nabla}_{2}\left(a+t_{0} b\right)=b^{2}-L^{*}\left(a, b, t_{0}, \varepsilon\right) .
\end{array}\right\}
$$

Solve the system:

$$
\left\{\begin{array}{l}
\text { (1) } v_{1}\left(a+t_{0} b, c_{1}, c_{2}\right)-\frac{a}{a^{*}\left(a, b, t_{0}, \varepsilon\right)}=0 \\
\text { (2) } v_{2}\left(a+t_{0} b, c_{1}, c_{2}\right)-b^{2}+L^{*}\left(a, b, t_{0}, \varepsilon\right)=0
\end{array}\right\}
$$

to get suitable $c_{1}=c_{1}(\varepsilon), c_{2}=c_{2}(\varepsilon)$.

Since $s_{1} \neq 0$ our system has nonzero Jacobian. We solve for $w_{1}(t, \varepsilon)$ and get the result. 
7 (b) GENERAL SOLUTIONS. Alternatively, eliminating $w_{2}$ from the Universal Cauchy-Kowalewski System we get

$$
X\left(w_{1}, t, \varepsilon, \lambda, c_{1}, c_{2}\right)=0
$$

where $c_{1}, c_{2}$ are constants.

The general solution of a specific equation is obtained as follows:

(1) Replace $\lambda$ by $G\left(w_{1}, t\right)$ in (7.1).

(2) Take the limit as $\varepsilon \rightarrow 0$ of the result.

$X$ is derived from $L$ and $K$ and is like the familiar differential quotient in generality.

\section{REFERENCES}

1. Garabedian, P. R. Partial Differential Equations, John Wiley, 1964.

2. 01msted, J. Advanced Calculus, Appleton-Century. 


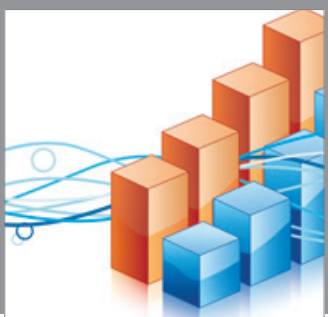

Advances in

Operations Research

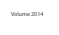

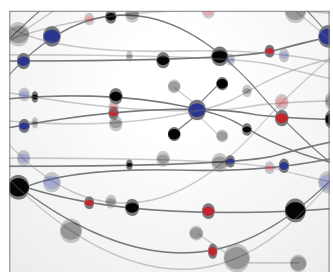

\section{The Scientific} World Journal
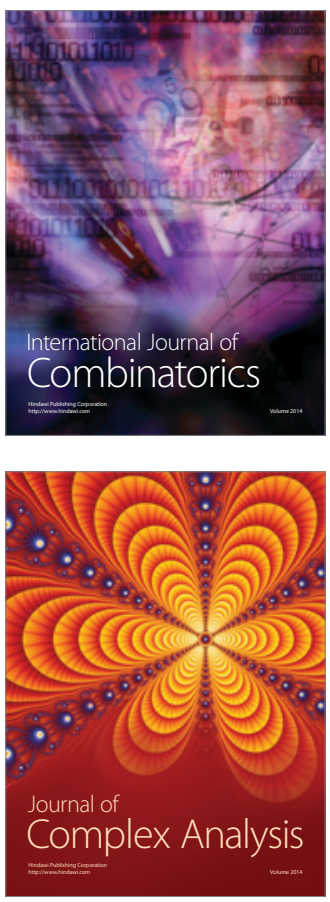

International Journal of

Mathematics and

Mathematical

Sciences
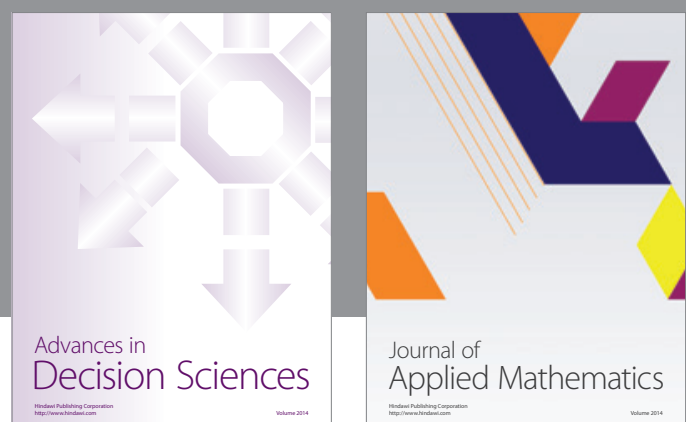

Journal of

Applied Mathematics
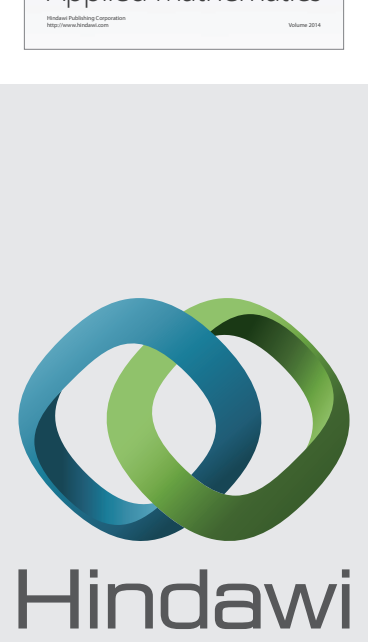

Submit your manuscripts at http://www.hindawi.com
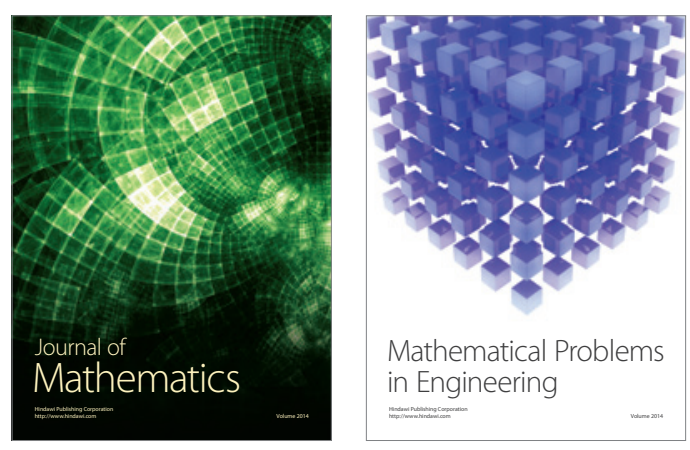

Mathematical Problems in Engineering
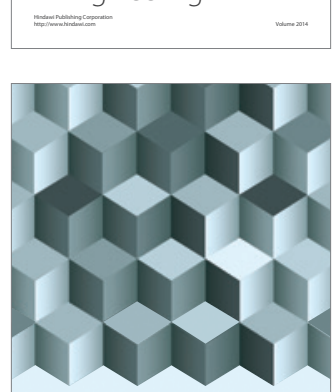

Journal of

Function Spaces
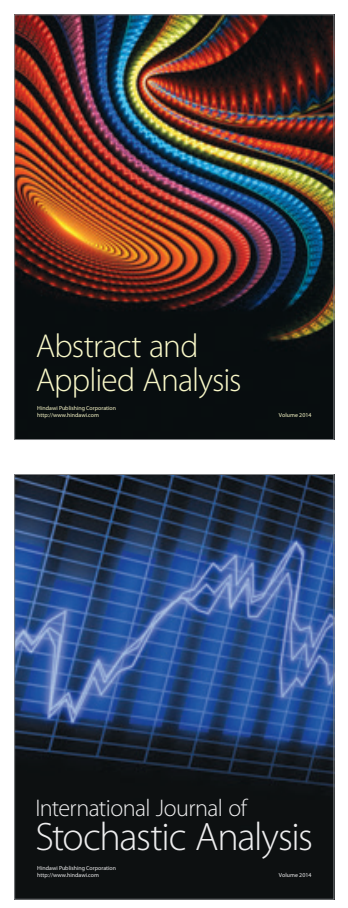

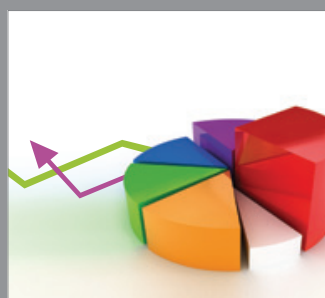

ournal of

Probability and Statistics

Promensencen
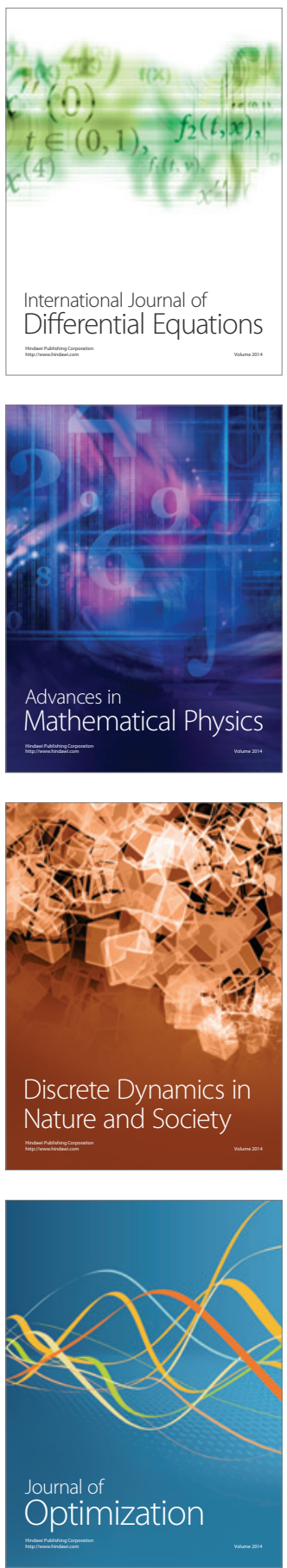